\title{
Warm wavebreaking of nonlinear plasma waves with arbitrary phase velocities
}

\author{
C. B. Schroeder, E. Esarey, and B. A. Shadwick \\ Lawrence Berkeley National Laboratory, \\ University of California, Berkeley, California 94720
}

(Dated: April 20, 2005)

\begin{abstract}
A warm, relativistic fluid theory of a nonequilibrium, collisionless plasma is developed to analyze nonlinear plasma waves excited by intense drive beams. The maximum amplitude and wavelength are calculated for nonrelativistic plasma temperatures and arbitrary plasma wave phase velocities. The maximum amplitude is shown to increase in the presence of a laser field. These results set a limit to the achievable gradient in plasma-based accelerators.
\end{abstract}

PACS numbers: 52.27.Ny, 52.38.-r, 52.38.Kd, 52.35.Mw 
Of fundamental interest in plasma physics are highly-nonlinear electron plasma waves, such as those produced in the laboratory via intense laser and beam plasma interactions [1]. Recent breakthrough results [2] on plasma-based accelerators have shown the production of high-quality electron bunches using ultra-high gradient $(\sim 100 \mathrm{GV} / \mathrm{m}$, several orders of magnitude beyond conventional technology) nonlinear plasma waves driven by intense laser pulses. In these experiments, the accelerated electrons were self-trapped from the background plasma through a process referred to as wavebreaking. A basic quantity of interest in plasma physics, and especially relevant to plasma accelerators, is the maximum plasma wave amplitude that is achievable prior to wavebreaking and the onset of self-trapping. Prior calculations [3-7] of the wavebreaking limit, however, are not valid in the regime of laser-plasma accelerator experiments.

In this Letter, a general result for the maximum field amplitude of a nonlinear electron plasma wave of arbitrary phase velocity in a warm plasma is derived from first principles. This result is valid in all regimes of interest, including that of short-pulse laser-plasma interactions, and reduce to the previous wavebreaking calculations [3-7] in the appropriate limits. The effects of an intense laser field are also included, as in the self-modulated regime of the laser wakefield accelerator [8-10], which is shown to increase the maximum field amplitude. The maximum field amplitude derived in this Letter sets the fundamental limit to the achievable gradient in plasma-based accelerators.

Using the cold, relativistic fluid equations in one-dimension (1D), the maximum electric field amplitude of a plasma wave was found [3] to be $E_{\mathrm{WB}}=\sqrt{2}\left(\gamma_{\varphi}-1\right)^{1 / 2} E_{0}$, which is referred to as the cold relativistic wavebreaking field. Here $\gamma_{\varphi}^{2}=1 /\left(1-\beta_{\varphi}^{2}\right)$ is the relativistic Lorentz factor, $v_{\varphi}=c \beta_{\varphi}$ is the plasma wave phase velocity, and $E_{0}=c m \omega_{p} / e$ is referred to as the nonrelativistic wavebreaking field, with $\omega_{p}=\left(4 \pi n_{0} e^{2} / m\right)^{1 / 2}$ the plasma frequency and $n_{0}$ the ambient electron plasma density. For a laser driven plasma wave, $v_{\varphi}$ is approximately the group velocity of the laser pulse, $\gamma_{\varphi} \simeq \omega_{0} / \omega_{p}$, where $\omega_{0}$ is the laser frequency. For a charged particle beam driver, $v_{\varphi}$ is approximately the particle beam velocity. When the plasma wave field approaches $E_{\mathrm{WB}}$, the cold plasma density becomes singular $n \rightarrow \infty[4]$. This singularity indicates a breakdown of the cold fluid equations.

Finite temperature fluid theories were applied to calculate the maximum amplitudes in the limits of nonrelativistic $\left(\gamma_{\varphi} \simeq 1\right)[5]$ and ultra-relativistic $\left(\beta_{\varphi}=1\right)[6,7]$ plasma waves. In the $\beta_{\varphi}=1$ limit, the maximum field was found $[6,7]$ to be $E_{\mathrm{th}}=\theta^{-1 / 4} \rho_{\mathrm{th}}\left(\gamma_{\varphi}, \theta\right) E_{0}$, where 
$\theta$ is the initial plasma temperature normalized to $m c^{2} / k_{\mathrm{B}}$, with $k_{\mathrm{B}}$ the Boltzmann constant, and $\rho_{\mathrm{th}}\left(\gamma_{\varphi}, \theta\right) \sim 1$ is a slowly-varying function of $\gamma_{\varphi}$ and $\theta$. This expression for $E_{\mathrm{th}}$ is valid for $\gamma_{\varphi} \theta^{1 / 2} \gg 1$, e.g., for an ultra-relativistic $\left(\beta_{\varphi}=1\right)$ particle beam driver. For laser-driven plasma waves, however, typically $\gamma_{\varphi} \sim 10-100$ and $\theta m c^{2} \sim 10 \mathrm{eV}[11,12]$. Therefore, a laser-plasma accelerator typically satisfies $\gamma_{\varphi} \theta^{1 / 2}<1$, and, hence, the above expression for $E_{\text {th }}$ does not apply. In addition, $E_{\text {th }}$ does not reduce to the nonrelativistic result [5] or the cold result $E_{\mathrm{WB}}$.

Standard warm relativistic fluid theories derived for collisionally-dominated plasmas (e.g., Ref. [13]) are inadequate for describing short-pulse laser-plasma interactions. Short-pulse laser-plasma interactions access a collisionless regime that is not in local thermodynamical equilibrium, in which the plasma electrons experience relativistic motion while the temperature (electron momentum spread) remains small. To model short-pulse laser-plasma interactions, we start with the covariant form of the collisionless Boltzmann equation [13],

$$
p^{\mu} \partial_{\mu} f-\left[\left(e / m c^{2}\right) F^{\alpha \nu} p_{\nu}\right] \partial f / \partial p^{\alpha}=0,
$$

where $f(\boldsymbol{x}, \boldsymbol{p}, t)$ is the phase space density, $x^{\mu}=(c t, \boldsymbol{x}), p^{\nu}=(\gamma, \gamma \boldsymbol{\beta})$ is the normalized particle four-momentum, $\partial^{\mu}=\left(\partial_{c t},-\nabla\right)$, and $F^{\mu \nu}=\partial^{\mu} A^{\nu}-\partial^{\nu} A^{\mu}$ is the electromagnetic fieldstrength tensor, with $A^{\mu}=(\Phi, \boldsymbol{A})$ the four-vector potential and $g^{\mu \nu}=\operatorname{diag}(1,-1,-1,-1)$ the space-time metric tensor.

We consider the following centered moments of the phase-space distribution [14-16]: $\Theta^{\mu \nu}=\int\left(p^{\mu}-u^{\mu}\right)\left(p^{\nu}-u^{\nu}\right) f d \Omega$ and $Q^{\alpha \mu \nu}=\int\left(p^{\alpha}-u^{\alpha}\right)\left(p^{\mu}-u^{\mu}\right)\left(p^{\nu}-u^{\nu}\right) f d \Omega$, where $u^{\mu}=J^{\mu} / h$ is the normalized hydrodynamic four-momentum, $h=\int f d \Omega$ the invariant particle density, $J^{\mu}=\int p^{\mu} f d \Omega$ the fluid four-current, and $d \Omega=d^{3} \boldsymbol{p} / p^{0}$ the Lorentz invariant momentumspace volume. Equation (1) implies the exact conservation laws

$$
\begin{gathered}
\partial_{\mu}\left(h u^{\mu}\right)=0, \\
h u^{\mu} \partial_{\mu} u^{\nu}+\partial_{\mu} \Theta^{\mu \nu}=\left(-e / m c^{2}\right) F^{\nu \alpha} h u_{\alpha}, \\
h u^{\alpha} \partial_{\alpha}\left(\Theta^{\mu \nu} / h\right)+\Theta^{\nu \alpha} \partial_{\alpha} u^{\mu}+\Theta^{\mu \alpha} \partial_{\alpha} u^{\nu}+\partial_{\alpha} Q^{\alpha \mu \nu} \\
=\left(-e / m c^{2}\right)\left(F^{\nu \alpha} \Theta_{\alpha}^{\mu}+F^{\mu \alpha} \Theta_{\alpha}^{\nu}\right),
\end{gathered}
$$

which correspond to the continuity equation, energy-momentum conservation, and energymomentum flux conservation, respectively. The inhomogeneous Maxwell equations are expressed as $\partial_{\mu} F^{\mu \nu}=4 \pi \sum_{s} q_{s} J_{s}^{\nu}$, where the sum is over species with $q$ the charge. 
We will assume a "warm" plasma such that the distribution $f$ has a small momentum spread about its mean [14-17]. We make no additional assumptions concerning the specific form of $f$. This warm assumption will allow the hierarchy of moment equations to be truncated. We define the invariant measure of thermal spread $\epsilon^{2}=-\Theta_{\mu}^{\mu} / h=u^{\mu} u_{\mu}-1$, where $|\epsilon| \ll 1$ is assumed, such that $\beta_{\mathrm{th}}^{2}=\epsilon^{2}\left(1+\epsilon^{2}\right)^{-1} \simeq \epsilon^{2}$ is the normalized thermal velocity spread (temperature). We will assume that in the local plasma rest frame $\Theta^{\mu \nu} / h=\mathcal{O}\left(\epsilon^{2}\right)$ and $Q^{\alpha \mu \nu} / h=\mathcal{O}\left(\epsilon^{3}\right)$. Truncation of the moment hierarchy to order $\mathcal{O}\left(\epsilon^{2}\right)$ is achieved by neglecting the third-order centered moment $Q^{\alpha \mu \nu}$ in the fluid equations. Note that $\epsilon$ is a Lorentz invariant and $\epsilon^{2} \ll 1$ is satisfied if the local rest frame temperature of the plasma is nonrelativistic. We consider the ratio $\lambda=n_{p} / h[14,16]$, where $n_{p}=\left(J^{\mu} J_{\mu}\right)^{1 / 2}$ is the proper density, and introduce $(\lambda \Gamma, \lambda \Gamma \boldsymbol{w})=u^{\mu}=J^{\mu} / h$, where $u^{\mu} u_{\mu}=\lambda^{2}$ and $\Gamma^{-2}=(1-\boldsymbol{w} \cdot \boldsymbol{w})$. Using the contraction of the energy-momentum stress tensor we find $\lambda^{2}=1-\Theta_{\mu}^{\mu} / h=1+\epsilon^{2}$, such that $\lambda=\left(1-\beta_{\mathrm{th}}^{2}\right)^{-1 / 2}$ is identified as the Lorentz factor associated with the thermal fluctuations.

Consider a plasma wave driven by a laser pulse propagating in the $z$-direction with transverse normalized vector potential $\boldsymbol{a}_{\perp}=e \boldsymbol{A}_{\perp} / m c^{2}$ (Coulomb gauge). We consider 1D motion such that $f=g\left(z, p_{z}, t\right) \delta^{2}\left(\boldsymbol{p}_{\perp}-\boldsymbol{a}_{\perp}\right)$ and the transverse component of Eq. (3) reduces to $u^{\mu} \partial_{\mu}\left(\lambda \Gamma \boldsymbol{w}_{\perp}-\boldsymbol{a}_{\perp}\right)=0$. For an initially quiescent $(\boldsymbol{w}=0)$ plasma, $\lambda \Gamma \boldsymbol{w}_{\perp}=\boldsymbol{a}_{\perp}$, i.e., $\boldsymbol{w}_{\perp}=\boldsymbol{a}_{\perp}\left(1-w_{z}^{2}\right)^{1 / 2}\left(\gamma_{\perp}^{2}+\epsilon^{2}\right)^{-1 / 2}$, with $\gamma_{\perp}=\left(1+a_{\perp}^{2}\right)^{1 / 2}$. This is the generalization of canonical transverse fluid momentum conservation including thermal effects.

The contraction $g_{\mu \nu} Q^{\alpha \mu \nu}=0$ [to order $\left.\mathcal{O}\left(\epsilon^{2}\right)\right]$ implies $\Theta^{\mu 0}=w_{z} \Theta^{\mu 1}$, and $\lambda^{2}=1+(1-$ $\left.w_{z}^{2}\right) \Theta^{11} / h$. Equations (2), (3), and (4) can be combined to yield

$$
u^{\mu} \partial_{\mu}\left(h^{-3} \Gamma^{-2} \Theta^{11}\right)=0 .
$$

For an initially quiescent plasma of density $n_{0}, \Theta^{11} / n_{0}=\Gamma^{2}\left(h / n_{0}\right)^{3} \theta$, where $\theta$ is the initial temperature normalized to $m c^{2} / k_{\mathrm{B}}$. Equation (5) is equivalent to a statement of entropy conservation.

Next, we assume the quasi-static approximation, such that the plasma wave driver (e.g., laser field or particle beam) and fluid quantities are functions only of $\xi=z-\beta_{\varphi} c t$. The continuity equation Eq. (2) becomes

$$
\partial_{\xi}\left[h \lambda \Gamma\left(\beta_{\varphi}-w_{z}\right)\right]=0,
$$


or, for an initially quiescent plasma of density $n_{0}, h=n_{0}\left[\lambda \Gamma\left(1-\beta_{\varphi}^{-1} w_{z}\right)\right]^{-1}$. The components of Eq. (3) can be combined to yield [using $\Theta^{\mu 0}=w_{z} \Theta^{\mu 1}$ and Eq. (6)]

$$
\partial_{\xi}\left[\left(h \lambda^{2} \Gamma^{2}+\Theta^{11}\right)\left(1-\beta_{\varphi} w_{z}\right)\left(1-\beta_{\varphi}^{-1} w_{z}\right)\right]=n_{0} \partial_{\xi} \phi
$$

where $\phi=e \Phi / m c^{2}$ is the normalized space-charge potential of the plasma wave and $(\lambda \Gamma)^{2}=$ $\left(\gamma_{\perp}^{2}+\epsilon^{2}\right) /\left(1-w_{z}^{2}\right)$. Using Eqs. (5) and (6), Eq. (7) can be written as the following longitudinal constant of motion (conservation of energy in the wave frame):

$$
\partial_{\xi}\left[\frac{\gamma_{\perp}\left(1-\beta_{\varphi} w_{z}\right)}{\left(1-w_{z}^{2}\right)^{1 / 2}}-\phi+\frac{3}{2} \theta \frac{\left(1-\beta_{\varphi} w_{z}\right)\left(1-w_{z}^{2}\right)^{1 / 2}}{\gamma_{\perp}\left(1-\beta_{\varphi}^{-1} w_{z}\right)^{2}}\right]=0
$$

The third term on the right-hand side of Eq. (8) is due to the energy in the thermal fluctuations (pressure).

The plasma wave potential is determined by the Poisson equation $c^{2} \partial_{\xi}^{2} \phi=\omega_{p}^{2}\left[J^{0} / n_{0}-\right.$ $\left.1+n_{b} / n_{0}\right]$, where $n_{b} / n_{0}$ is the normalized density of a beam driver, $J^{0} / n_{0}=\lambda \Gamma h / n_{0}=$ $\beta_{\varphi} /\left(\beta_{\varphi}-w_{z}\right)$, and the ions are assumed stationary. The Poisson equation can be combined with Eq. (8) to yield the evolution equation for the axial plasma fluid velocity $w_{z}$.

We consider the cases of plasma wave excitation behind a beam driver where $n_{b}(\xi)=$ 0, behind a short laser driver (e.g., the standard laser wakefield regime) where $\gamma_{\perp}=1$, and excitation under a long laser pulse (e.g., the self-modulated laser wakefield regime) where $\gamma_{\perp}^{-1}\left|\left(c / \omega_{p}\right) \partial_{\xi} \gamma_{\perp}\right| \ll 1$ and $\gamma_{\perp} \simeq$ constant. Using Eq. (8), the first integral of the Poisson equation is (assuming $n_{b}=0$ and $\gamma_{\perp}=$ constant) $\hat{E}^{2}=\gamma_{\perp}\left(\chi_{0}-\chi+\chi_{0}^{-1}-\chi^{-1}\right)+$ $\left[F\left(\chi_{0}\right)-F(\chi)\right] \theta / \gamma_{\perp}$, where $\chi^{2}=\left(1-w_{z}\right) /\left(1+w_{z}\right), \hat{E}=E / E_{0}=-\left(c / \omega_{p}\right) \partial_{\xi} \phi(w)$,

$$
F(\chi)=\frac{6 \beta_{\varphi}^{2} \chi\left[\left(1-\chi^{4}\right)-\beta_{\varphi}\left(\chi^{4}-2 \chi^{2} / 3+1\right)\right]}{\left[\left(1-\beta_{\varphi}\right)-\left(1+\beta_{\varphi}\right) \chi^{2}\right]^{3}},
$$

and $\chi_{0}$ corresponds to the momentum that produces the extremum of $\phi$ [i.e., $\left.\hat{E}\left(\chi_{0}\right)=0\right]$.

Solving $\partial_{\xi} \phi=0$ (i.e., a quartic equation for $\chi_{0}^{2}$ ), yields the momentum which produces the extremum of $\phi$,

$$
\begin{aligned}
\chi_{0}^{2} & =\gamma_{\varphi}^{2}\left(1-\beta_{\varphi}\right)^{2}+\frac{1}{2} \gamma_{\perp}^{-2}\left(1+\beta_{\varphi}\right)^{-2}\left\{3 \beta_{\varphi}^{2} \theta+\beta_{\varphi}\left(48 \theta \gamma_{\perp}^{2} / \gamma_{\varphi}^{2}+9 \beta_{\varphi}^{2} \theta^{2}\right)^{1 / 2}\right. \\
& \left.+\left[6 \theta \beta_{\varphi}^{2}\left(10 \gamma_{\perp}^{2} / \gamma_{\varphi}^{2}+3 \beta_{\varphi}^{2} \theta\right)+2 \beta_{\varphi}\left(2 \gamma_{\perp}^{2} / \gamma_{\varphi}^{2}+3 \beta_{\varphi}^{2} \theta\right)\left(48 \theta \gamma_{\perp}^{2} / \gamma_{\varphi}^{2}+9 \beta_{\varphi}^{2} \theta^{2}\right)^{1 / 2}\right]^{1 / 2}\right\} .
\end{aligned}
$$

Equation (10) determines the fluid momentum at the maximum compression of the plasma. In the cold limit $(\theta=0), \chi_{0}^{2}=\gamma_{\varphi}^{2}\left(1-\beta_{\varphi}\right)^{2}$ and the extremum of the potential occurs when 
the axial fluid velocity equals the phase velocity of the wave, i.e., $w_{z}=\beta_{\varphi}$. In the ultra-high phase velocity limit $\left(\beta_{\varphi}=1\right), \chi_{0}^{2}=3 \gamma_{\perp}^{-2} \theta / 2$.

Using the Poisson equation, the phase where $\hat{E}$ is maximum $\left(\partial_{\xi} \hat{E}=0\right)$ occurs at the momentum $\chi=1$ (i.e., $w_{z}=0$ ). Evaluating $\hat{E}^{2}$ at $\chi=1$ yields

$$
\hat{E}_{\max }^{2}=\gamma_{\perp}\left(\chi_{0}+\chi_{0}^{-1}-2\right)+\left[F\left(\chi_{0}\right)-1\right] \theta / \gamma_{\perp}
$$

where $F\left(\chi_{0}\right)$ is given by Eqs. (9) and (10). Equation (11) is the main result of this Letter, and determines the maximum field amplitude $E_{\max }$ of a nonlinear plasma wave with phase velocity $\beta_{\varphi}$ excited in a plasma with initial temperature $\theta$. The maximum density perturbation is given by $\left(J^{0} / n_{0}\right)_{\max }=\left[1-\beta_{\varphi}^{-1}\left(1-\chi_{0}^{2}\right) /\left(1+\chi_{0}^{2}\right)\right]^{-1}$. Note that the maximum plasma density perturbation in a warm plasma does not become singular, as in the cold fluid theory $[3,4]$.

In the cold plasma limit $(\theta=0)$, Eq. (11) reduces to $\hat{E}_{\max }^{2}(\theta=0)=2 \gamma_{\perp}\left(\gamma_{\varphi}-1\right)$. This is a generalization of the cold relativistic wavebreaking field [3] to include a laser field. Note that $\hat{E}_{\max }^{2}(\theta=0)$ is the same as the threshold field for trapping background plasma electrons in a cold plasma wave [18] (since the cold fluid element orbits are identical to the particle orbits).

For $\beta_{\varphi} \ll 1$, Eq. (11) reduces to

$$
\frac{\hat{E}_{\max }^{2}}{\gamma_{\perp} \beta_{\varphi}^{2}} \simeq 1-\frac{8}{3}\left(\frac{3 \theta}{\gamma_{\perp}^{2} \beta_{\varphi}^{2}}\right)^{1 / 4}+2\left(\frac{3 \theta}{\gamma_{\perp}^{2} \beta_{\varphi}^{2}}\right)^{1 / 2}-\frac{1}{3}\left(\frac{3 \theta}{\gamma_{\perp}^{2} \beta_{\varphi}^{2}}\right)
$$

where terms of order $\mathcal{O}\left(\theta \beta_{\varphi}^{2}\right)$ have been neglected. For $\gamma_{\perp}=1$, Eq. (12) is identical to the result of Ref. [5].

For $\beta_{\varphi}=1$ (e.g., an ultra-relativistic electron beam driver satisfying $\gamma_{\varphi}^{-2} \ll \theta \ll 1$ ), Eq. (11) reduces to

$$
\hat{E}_{\max }^{2}=\gamma_{\perp}^{2}(2 / 3)^{3 / 2} \theta^{-1 / 2}\left[1-\gamma_{\perp}^{-1}(3 \theta / 2)^{1 / 2}\right]^{3}
$$

For the case $\gamma_{\perp}=1$, Eq. (13) scales to leading order as $E_{\max }=\theta^{-1 / 4} \rho_{\mathrm{th}} E_{0}$. Except for the factor $\rho_{\text {th }} \sim 1$, this scaling is the same as that obtained in Refs. [6, 7].

In the limit, $\theta \ll \gamma_{\perp}^{2} / \gamma_{\varphi}^{2} \ll 1$, Eq. (11) reduces to

$$
\hat{E}_{\max }^{2} \simeq 2 \gamma_{\perp}\left(\gamma_{\varphi}-1\right)-2 \gamma_{\varphi}\left[\frac{4}{3}\left(3 \gamma_{\varphi}^{2} \gamma_{\perp}^{2} \theta\right)^{1 / 4}-\left(3 \gamma_{\varphi}^{2} \theta\right)^{1 / 2}\right]
$$


Equation (14) is the cold relativistic wavebreaking field (generalized to include a laser field) with the lowest order reduction due to the plasma temperature. For high-intensity lasers $\left(a_{\perp} \gtrsim 1\right)$, Eq. (14) indicates that $E_{\max }$ inside a laser pulse is significantly larger compared to behind the pulse (where $a_{\perp}=0$ ). For a laser-driver, the phase velocity of the plasma wave is approximately the nonlinear group velocity of the laser pulse, i.e., $\gamma_{\varphi} \simeq\left[\gamma_{\perp}(1+\right.$ $\left.\left.\gamma_{\perp}\right) / 2\right]^{1 / 2}\left(\omega_{0} / \omega_{p}\right)$. Therefore, for ultra-high intensities $\left(a_{\perp} \gg 1\right), \hat{E}_{\max } \simeq\left(2 \gamma_{\perp} \gamma_{\varphi}\right)^{1 / 2} \sim$ $a_{\perp}\left(\omega_{0} / \omega_{p}\right)^{1 / 2}$ in the limit $\theta \ll \gamma_{\perp}^{2} / \gamma_{\varphi}^{2} \ll 1$.

The transition from the laser-driven regime $\left(\gamma_{\varphi}^{2} \theta<1\right)$ to the ultra-relativistic beamdriven regime $\left(\gamma_{\varphi}^{2} \theta \gg 1\right)$ is shown if Fig. 1, which plots $\hat{E}_{\max }$ [Eq. (11)] versus $\gamma_{\varphi}^{2} \theta$ for $\theta=10^{-3}, \theta=10^{-4}$, and $\theta=10^{-5}$ with $\gamma_{\perp}=1$. The dashed lines in Fig. 1 are the $\beta_{\varphi}=1$ limit [Eq. (13)]. Note that for typical short-pulse laser-plasma-interactions, $\theta m c^{2} \sim 10 \mathrm{eV}$ $[11,12]$, or $\theta \sim 5 \times 10^{-5}$. Figure 1 shows the inaccuracy of using the ultra-high phase velocity approximation $\left(\beta_{\varphi}=1\right)$ in the laser-plasma accelerator parameter regime $\left(\theta \gamma_{\varphi}^{2}<1\right)$.

The wavelength $\lambda_{\text {osc }}$ of the nonlinear plasma oscillation at the maximum amplitude is computed from $\hat{E}$ by $\lambda_{\text {osc }}=\int d \xi=-2 c \omega_{p}^{-1} \int(d \phi / d \chi) \hat{E}^{-1} d \chi$ between the extrema of $\chi$. Figure 2 shows the wavelength of the plasma oscillation $\lambda_{\text {osc }}$ normalized to $\lambda_{p}=2 \pi c / \omega_{p}$ versus $\gamma_{\varphi}$ for initial temperatures $\theta=10^{-3}, \theta=10^{-4}$, and $\theta=10^{-5}$, with $\gamma_{\perp}=1$. The dashed line in Fig. 2 shows $\lambda_{\text {osc }} / \lambda_{p}$ for an initially cold plasma $\theta=0$.

The temperature (thermal velocity spread) evolution is given by $\beta_{\mathrm{th}}^{2}=\epsilon^{2}=\theta(1-$ $\left.w_{z}^{2}\right) \Gamma^{2}\left(h / n_{0}\right)^{2}$, which is maximum at the maximum compression of the plasma $\left(\chi=\chi_{0}\right)$, i.e., $\epsilon_{\max }^{2}=4 \chi_{0}^{2}\left[\left(1+\chi_{0}^{2}\right)-\beta_{\varphi}^{-1}\left(1-\chi_{0}^{2}\right)\right]^{-2} \theta$. For an ultra-relativistic beam driver $\left(\beta_{\varphi}=1\right.$ and $\left.\gamma_{\perp}=1\right), \epsilon_{\max }^{2}=2 / 3$ [the upper bound of $\epsilon_{\max }^{2}\left(\beta_{\varphi}\right)$ ]. In the limit $\theta \ll \gamma_{\perp}^{2} / \gamma_{\varphi}^{2} \ll 1$ (e.g., laser driver), the maximum temperature is, to leading order, $\epsilon_{\max }^{2} \simeq \gamma_{\perp}\left(\gamma_{\varphi}^{2} \theta / 3\right)^{1 / 2}[1-$ $\left.\left(3 \gamma_{\varphi}^{2} \theta\right)^{1 / 2} /\left(4 \gamma_{\perp}\right)\right] \ll 1$, which confirms the validity of the warm plasma approximation $\epsilon^{2} \ll 1$.

The above results for $\hat{E}_{\max }$ are independent of the driver. Consider excitation by a laser pulse with length optimized to maximize the wave amplitude. As the laser intensity increases, the wave amplitude increases, up to the amplitude at which $|\hat{E}|=\hat{E}_{\max }$, which is first reached behind the laser pulse (where $\gamma_{\perp}=1$ ). Note that the maximum density compression occurs at the phase where $\hat{E}=0$, which is at a phase behind that where $|\hat{E}|=\hat{E}_{\max }$ in a warm plasma. Physically, the limit on the wave amplitude is due to the pressure force. As the plasma becomes highly compressed, the pressure force grows, ultimately limiting the density compression and therefore the wave amplitude. This is in 
contrast to cold fluid theories where the maximum field is reached when the density becomes singular (and shock formation occurs). For larger drive intensities, no force balance is possible, and no travelling wave solutions exist. Further calculations indicate that $\hat{E}=\hat{E}_{\max }$ corresponds to significant trapping of the electrons in the wave [19].

For a laser with a square pulse profile, the maximum amplitude is obtained when the laser pulse length is of an optimal value such that $\partial_{\xi} \phi=0$ at the end of the laser pulse. Note that, for an optimal length driver, the laser initially reduces the plasma density and the pressure force will remain small during the excitation of the plasma wave by the laser pulse. For relativistic plasma waves $\left(\gamma_{\varphi}^{2} \gg 1\right)$, the laser intensity required to excite the maximum field Eq. (11) is $\gamma_{\perp} \simeq \hat{E}_{\max } / 2+\left[\left(\hat{E}_{\max } / 2\right)^{2}+1\right]^{1 / 2}$. The limits $\gamma_{\varphi}^{2} \gg 1$ and $\gamma_{\varphi}^{2} \theta \ll 1$, imply $\gamma_{\perp} \simeq \sqrt{2 \gamma_{\varphi}}\left[1-\left(2^{3 / 2} / 3\right)\left(\gamma_{\varphi}^{2} \theta / 2\right)^{1 / 4}+(5 / 9)\left(\gamma_{\varphi}^{2} \theta / 2\right)^{1 / 2}\right]$.

In this Letter, a comprehensive theory has been presented that describes the properties of nonlinear electron plasma waves with arbitrary phase velocity in a warm plasma, including the presence of an intense laser field. An analytical result for the maximum field amplitude is derived, Eq. (11). Equation (11) is capable of describing the regime of current ultra-intense short-pulse laser interactions with underdense plasma, in contrast to previous results that are limited to ultra-relativistic particle drive beams. The maximum field is larger in the presence of an intense laser field. These results place a fundamental limit on the accelerating gradient in plasma-based accelerators.

The authors gratefully acknowledge discussions with A. Charman. This work was supported by the Office of Science, High Energy Physics, U.S. Department of Energy under Contract No. DE-AC03-76SF0098. 
[1] E. Esarey et al., IEEE Trans. Plasma Sci. 24, 252 (1996).

[2] S. P. D. Mangles et al., Nature 431, 535 (2004); C. G. R. Geddes et al., Nature 431538 (2004); Faure et al., Nature 431, 541 (2004).

[3] A. I. Akhiezer and R. V. Polovin, Zh. Eksp. Teor. Fiz. 30, 915 (1956).

[4] J. M. Dawson, Phys. Rev. 113, 383 (1959).

[5] T. P. Coffey, Phys. Fluids 14, 1402 (1971).

[6] T. Katsouleas and W. B. Mori, Phys. Rev. Lett. 61, 90 (1988).

[7] J. B. Rosenzweig, Phys. Rev. A 38, 3634 (1988).

[8] A. Modena et al., Nature 377, 606 (1995).

[9] D. Umstadter et al., Science 273, 472 (1996).

[10] W. P. Leemans et al., Phys. Rev. Lett. 89, 174802 (2002).

[11] C. G. Durfee III, J. Lynch, and H. M. Milchberg, Phys. Rev. E 51, 2368 (1995).

[12] P. Volfbeyn, E. Esarey, and W. Leemans, Phys. Plasmas 6, 2269 (1999).

[13] S. R. de Groot, W. A. van Leeuwen, and C. G. van Weert, Relativistic Kinetic Theory (NorthHolland, Amsterdam, 1980).

[14] W. A. Newcomb, Phys. Fluids 25, 846 (1982).

[15] P. Amendt, Phys. Fluids 29, 1458 (1986).

[16] J. G. Siambis, Phys. Fluids 30, 896 (1986).

[17] B. A. Shadwick, G. M. Tarkenton, and E. H. Esarey, Phys. Rev. Lett. 93, 175002 (2004).

[18] E. Esarey et al., Phys. Plasmas 6, 2262 (1999).

[19] C. B. Schroeder et al., in Advanced Accelerator Concepts, edited by V. Yakimenko (AIP, New York, 2004), pp. 564-570. 


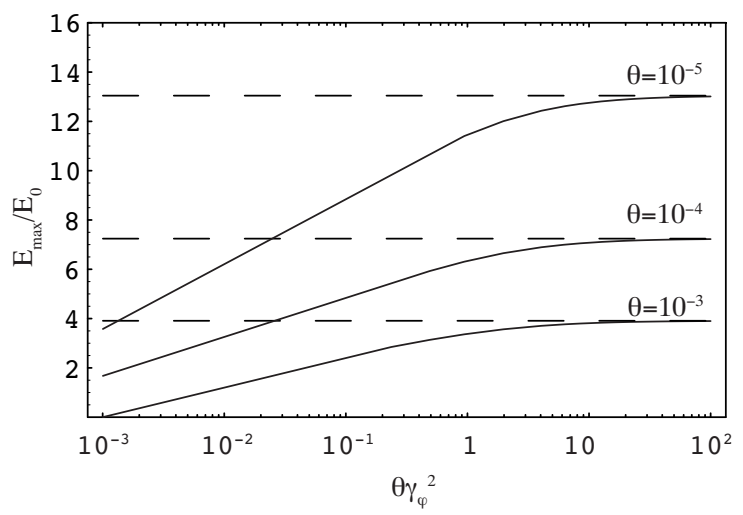

FIG. 1: Maximum plasma wave electric field $\hat{E}_{\max }=E_{\max } / E_{0}$ [Eq. (11)] versus $\theta \gamma_{\varphi}^{2}$ for initial temperatures $\theta=10^{-3}, \theta=10^{-4}$, and $\theta=10^{-5}$, with $\gamma_{\perp}=1$. Dashed lines are the ultra-high phase velocity result $\beta_{\varphi}=1$ [Eq. (13)].

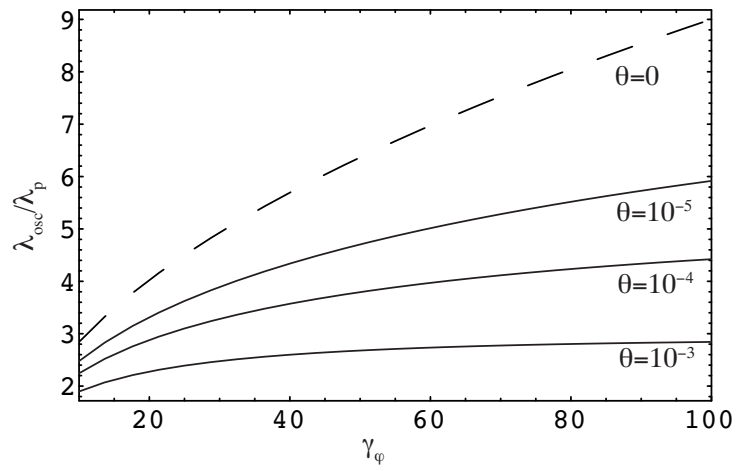

FIG. 2: Normalized nonlinear plasma wavelength $\lambda_{\text {osc }} / \lambda_{p}$ versus $\gamma_{\varphi}$ for initial temperatures $\theta=$ $10^{-3}, \theta=10^{-4}$, and $\theta=10^{-5}$, with $\gamma_{\perp}=1$. Dashed line is the cold limit $\theta=0$. 